\title{
Participación de la Policía Nacional en Misiones de Paz de la OnU y sus beneficios para el posconflicto ${ }^{1}$
}

\author{
https://doi.org/10.21830/9789585380219.04
}

\begin{abstract}
Aureliano Sánchez Mejía ${ }^{2}$
Steven Aldemar Mora Garzón ${ }^{3}$
\end{abstract}

Escuela de Cadetes de Policía "General Francisco de Paula Santander"

Resumen. Esta investigación identifica los beneficios que obtiene la Policía Nacional por participar en las Misiones de Paz de la Organización de las Naciones Unidas (ONU) y su efecto en el posconflicto colombiano, así como el aporte que hacen sus uniformados a dichas misiones gracias a la experiencia que ha obtenido la institución en sus más de 128 años de historia. Con este propósito se implementó una metodología de tipo cualitativa, que estuvo fundamentada en las bases teóricas y empíricas recopiladas durante la investigación. Para obtener la información se realizó una encuesta semiestructurada a los uniformados activos de la Policía Nacional que han participado en las Misiones de Paz de la ONU, con respecto al conocimiento y las habilidades de tipo social, humanístico, táctico u operacional que hayan adquirido o aportado en dichas misiones. Los resultados muestran que la participación activa de los uniformados de la Policía Nacional en estas misiones fortalece los lazos internacionales y amplía el conocimiento recíproco entre la institución y los organismos humanitarios debido a su interacción en los conflictos externos para el restablecimiento de la paz, lo cual contribuye en gran medida a la implementación de

1 Este capítulo forma parte de los resultados del proyecto de investigación "Educación policial", del grupo de investigación Ecsan de la Escuela de Cadetes de Policía "General Francisco de Paula Santander”, categorizado en C por Minciencias y con código de registro COL0080809. Los puntos de vista que se presentan en este capítulo pertenecen a los autores y no reflejan necesariamente los de las instituciones participantes.

2 Subteniente de la Policía Nacional de Colombia. Magíster en Ciencias Económicas y Empresariales. Especialista en Desarrollo Económico Regional y especialista en Servicio de Policía (EcSAN). Economista de la Universidad de Pamplona. Coordinador de Investigaciones en el Centro de Estudios Competitics y docente de la Universidad de Investigación y Desarrollo, Bucaramanga, Colombia. OrCID: https://orcid.org/0000-0001-6398-1975 - Contacto: aa.sanchez00055@policia.edu.co

3 Subteniente de la Escuela de Cadetes “General Francisco de Paula Santander”. Abogado de la Universidad Nacional de Colombia. Especialista (c) en Servicio de Policía (EcSAN). Pasante investigador del Programa de la Participación Misiones Internacionales por parte de las Fuerzas Militares de Colombia (Ejército Nacional de Colombia). Orcid: https://orcid.org/0000-0003-2418-7140 - Contacto: steven.mora@ policia.edu.co 
protocolos en el marco del posconflicto en Colombia para consolidar una paz duradera y estable en el territorio nacional.

Palabras clave: Derechos Humanos; Misiones de Paz; Policía Nacional; posconflicto; Organización de las Naciones Unidas; transferencia de conocimiento.

\section{Introducción}

Respecto a su proyección internacional, Colombia ha enfocado su relacionamiento internacional en diferentes frentes, entre los cuales se encuentra la participación de la Policía Nacional en las Misiones de Mantenimiento de Paz de la Organización de las Naciones Unidas (ONU). Es por ello que esta investigación pretende establecer las bases fundamentales que soportan la importancia de estas misiones para el fortalecimiento de la política exterior con respecto a la cooperación internacional. Específicamente, este estudio busca responder la siguiente pregunta: ¿Cuáles son los beneficios para el posconflicto en Colombia que se derivan de la participación de la Policía Nacional en las Misiones de Paz de la Onu?

Dado que Colombia cumple más con el requisito de país receptor de las Misiones de Paz de la ONu, que de país emisor de efectivos policiales, es necesario producir un documento que dé soporte académico y científico, con evidencia teórica y empírica, a los beneficios que trae consigo la participación de la Policía Nacional en las Misiones de Paz de la Onu y su aporte en los distintos ámbitos del posconflicto en Colombia.

Teniendo en cuenta lo anterior, es indispensable comenzar este trabajo conceptualizando las Misiones de Paz como uno de los mecanismo de mayor importancia con los que cuenta la ONU para cumplir su génesis misional de "prevenir y eliminar amenazas a la paz, y suprimir actos de agresión u otros quebrantamientos de la paz a partir de medios pacíficos, y de conformidad con los principios de la justicia y de derecho internacional" (Carta de las Naciones Unidas, art. 1). Asimismo, se debe tener presente que la paz es la base primordial para lograr el desarrollo económico de un país (Fajardo, 2017), ya que hace posible establecer una mayor destinación de recursos económicos para el fortalecimiento del talento humano local, lo cual permite la gestación y el desa- 
rrollo de una cadena productiva más fuerte y con mayor eficiencia (Lombana et al., 2018).

La evolución de la OnU ha proporcionado a las Misiones de Paz un horizonte histórico en el que han surgido "conceptos como la diplomacia preventiva y el establecimiento de la paz, el mantenimiento de la paz, la consolidación de la paz y, como última ratio, la imposición de la paz” (Vallejo, 2015, p. 39). De igual manera, cabe señalar que existen dos etapas que exponen la capacidad de actuación de las Misiones de Paz: una se refiere a las misiones que se desarrollaron durante la Guerra Fría, y la otra a todas las misiones siguientes (Boutros-Ghali, 1993, p. 1).

La evolución de las Misiones de Paz de la Onu ha establecido como prioridad el fomento del desarrollo humano en los territorios intervenidos como requisito primordial de paz (ONU, 2008, pp. 11-12), es por ello que se han delegado funciones a la policía de las Naciones Unidas, que ha pasado de ser simple observadora a participar de manera directa en la reconstrucción y la capacitación de las fuerzas policiales de los países receptores. En este sentido, la Onu (2016) establece que la "Policía [...] tiene la obligación de respetar y proteger los Derechos Humanos. Esta interpretación [...] debe ser el denominador común para los agentes de policía de las Naciones Unidas [...] con diversos marcos jurídicos" (p. 3).

Es importante resaltar que la Policía Nacional de Colombia ha estado participando en las Misiones de Paz de la Onu desde el año 1992, lo cual le ha permitido a sus uniformados obtener múltiples ópticas del conflicto armado y de las distintas rutas de intervención de la ONU de acuerdo con los diferentes entornos multiculturales y geopolíticos, como en los casos de "Camboya (1992 con el Apronuc), Yugoslavia (1992 Unprofor), El Salvador (1993 OnusaL), Guatemala (1997 Minugua), Haití (2006-2017 Minusth), Guinea-Bissau (2009-2016 Uniogbis) y Sierra Leona (2000 Unipsil)” (Policía Nacional, 2010, pp. 38-39).

La experiencia que ha ganado la Policía Nacional en las Misiones de Paz de la Onu le da la capacidad de generar valor agregado a su labor policial, con un énfasis en el relacionamiento con las comunidades y sus actores activos, en pro de crear espacios y mecanismos que permitan al Estado colombiano arti- 
cular "el sector público y privado, paralelo a los diálogos de paz, y trabajar en desarrollar estrategias que permitan adelantar acciones claras y efectivas para enfrentar los problemas de empleo, inseguridad y violencia que se viven actualmente en Colombia” (Rodríguez \& Suárez, 2017).

De igual manera, la participación de la Policía Nacional en las Misiones de Paz de la ONu ha fortalecido la imagen institucional en el exterior, gracias a las altas capacidades tácticas y operacionales que ha demostrado la institución en sus intervenciones en distintos países, las cuales se deben a la experiencia adquirida durante tantos ańos de lucha en contra de la insurgencia, el narcotráfico y el terrorismo. Es de esta manera que se revalida "como los observadores militares conceptúan que esta participación internacional permite una beneficiosa transferencia de conocimientos y la posibilidad de recibir cooperación por reciprocidad en caso de ser necesaria" (Pastrana, 2019, p. 358). Por esta razón es importante evaluar las competencias académicas y profesionales de los uniformados que la Policía Nacional selecciona para participar en las Misiones de Paz, "mediante la construcción de una matriz de diagnóstico del perfil ocupacional requerido por Naciones Unidas" (Álvarez, 2019, p. 86), de manera que dichas participaciones logren ser cada vez más destacadas.

Finalmente, es clara la existencia de una base teórica que permite soportar en gran medida la participación que ha tenido la Policía Nacional en las Misiones de Paz durante los 28 ańos de historia de cooperación internacional con la ONu. Sin embargo, también es fundamental establecer la evidencia empírica y sustancial tanto del nivel de conocimiento adquirido como del aportado y sus beneficios en pro de la labor de la Policía Nacional en los diferentes ámbitos del posconflicto en Colombia.

\section{Metodología}

\section{Diseño}

Los estudios científicos tienen como objetivo primordial describir las circunstancias y los acontecimientos de la forma más apegada y precisa a la realidad a partir de los factores causales (Babbie, 2000, p. 74). Teniendo esto en cuenta, se determinó realizar esta investigación a partir de la recolección 
documental del material existente del tema objeto de estudio y analizarlo mediante un método de carácter cualitativo. Este modelo permite al investigador fundamentar expresiones descriptivas de la realidad y relacionarlas con las diferentes interacciones del evento analizado, lo cual hace posible ofrecer una interpretación coherente de los beneficios de la participación de la Policía Nacional en las Misiones de Paz de la OnU.

\section{Instrumentos}

Esta investigación es de tipo documental, debido a que en este se utilizan distintos documentos impresos, audiovisuales o electrónicos como insumo principal (Morales, 2016, p. 2). De igual manera, se usó la investigación de campo, ya que a través de ella "se puede recurrir a otras fuentes distintas a las bibliográficas como, por ejemplo, el testimonio de los hechos, de testigos calificados" (Kaufman \& Rodríguez, 1993, p. 43). En este sentido, se aplicó una encuesta a los uniformados que han participado en las Misiones de Paz de la ONu y que actualmente permanecen activos en la Policía Nacional.

De esta manera fue posible obtener una buena proporción de material de datos primarios y secundarios a partir de diferentes métodos de recolección, como la encuesta mediante correo electrónico para los datos primarios y el análisis de documentos físicos y digitales de archivos bibliográficos para el caso de los datos secundarios.

\section{Universo, población objetivo y muestra}

Para la recopilación de los datos primarios mediante la encuesta propuesta, se proyectó en principio aplicarla a una muestra resultante de un muestreo probabilístico estándar, donde se estableciera un nivel de confianza del 95\%, con un margen de error del $5 \%$, como se evidencia en la ecuación 1 y en la tabla 1 .

$$
\mathrm{n}=\frac{N * Z^{2} * p * q}{\sigma^{2}(N-1)+Z^{2} * p * q} \quad \text { Ecuación } 1
$$


Tabla 1. Abreviatura estadística de la muestra

\begin{tabular}{ccl}
\hline Abreviatura & Valor & \multicolumn{1}{c}{ Definición } \\
\hline N & 70 & Población objetivo \\
Z & $95 \%$ & Nivel de confianza $(1,96)$ \\
P & $50 \%$ & Probabilidad de éxito $(0,5)$ \\
Q & $50 \%$ & Probabilidad de fracaso $(0,5)$ \\
$\sigma$ & $5 \%$ & Error $(0,05)$ \\
N & 59 & Muestra \\
\hline
\end{tabular}

Fuente: Cálculos propios a partir de información estadística necesaria para muestreo.

Para establecer la muestra de esta investigación se partió de un universo estadístico que tuvo en cuenta los 847 uniformados de la Policía Nacional que han sido parte de alguna de la Misiones de Paz de la OnU en toda su historia. De igual manera, se determinó como población objetivo a 70 uniformados que han participado en las Misiones de Paz y permanecen activos en la Policía Nacional. Ahora bien, dado que la fórmula estadística de muestreo determinó que la encuesta se debía aplicar a una muestra de 59 personas y que esta cifra era cercana a la población objetivo, entonces se determinó realizar la encuesta a la totalidad de la población objetivo, con el propósito de obtener resultados con un nivel de confianza del $100 \%$ sin margen de error.

\section{Análisis de los datos}

Para el análisis de los datos se tuvo en cuenta toda la información recolectada y producida mediante las diferentes técnicas e instrumentos utilizados durante la investigación, en la cual se tuvieron en cuenta los hechos históricos documentados para realizar el análisis descriptivo.

De igual forma, se realizó un proceso de estructuración y clasificación semántica de las categorías más relevantes que se hallaron en los datos cualitativos recopilados mediante las encuestas, los cuales fueron tabulados y estructurados en el software Microsoft Excel. Esto permitió que la interpretación de los resultados fuera clara y coherente con respecto a los beneficios que tiene la participación de la Policía Nacional en las Misiones de Paz de la OnU. 


\section{Resultados}

Durante el transcurso de esta investigación se analizó la interacción semántica de diferentes características comunes en las respuestas que se obtuvieron y su aporte a las diferentes categorías priorizadas como bases de beneficio internacional, nacional, institucional y personal de la participación de la Policía Nacional en las Misiones de Paz de la OnU.

De igual manera, se analizaron detenidamente los componentes humanísticos, sociales, tácticos y estratégicos, con el propósito de descubrir el nivel de capacidades y conocimiento que logra aportar o adquirir la Policía Nacional mediante su participación en dichas misiones. Esta investigación también permitió analizar el nivel de conocimiento que adquieren los miembros de la Policía Nacional en algunos componentes sociales esenciales en la labor policial en el marco del posconflicto (figura 1).

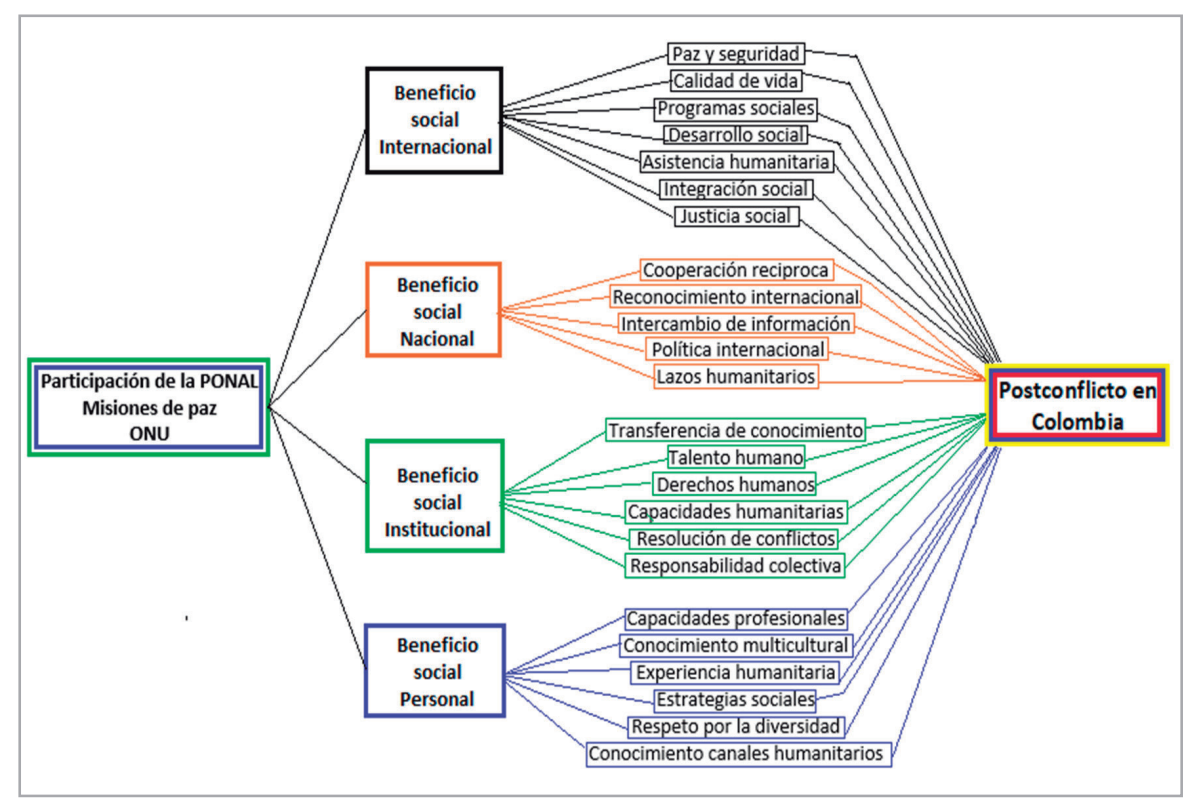

Figura 1. Beneficio social adquirido por la Policía Nacional en distintos ámbitos derivado de su participación en las Misiones de Paz de la ONU.

Fuente: Elaboración propia. 
Teniendo en cuenta la articulación semántica de la figura 1, la cual parte de las respuestas de los uniformados activos de la Policía Nacional que han participado en las Misiones de Paz de la OnU, es preciso establecer que el conjunto de interacciones existentes en los diferentes conjuntos de beneficios sociales priorizan la paz y la seguridad, al igual que la calidad de vida y los programas sociales, como los aspectos más relevantes en el ámbito internacional al que acceden los países que participan en estas misiones.

Con respecto al conjunto nacional, cabe resaltar que la priorización de las interacciones de mayor relevancia está determinada por la cooperación recíproca, el reconocimiento internacional de las altas capacidades de la Policía Nacional y el intercambio de información como beneficio nacional. Asimismo, se da relevancia a la política internacional y a los lazos humanitarios que se logra adquirir mediante la participación en las Misiones de Paz de la OnU.

En este mismo sentido, como institución, la Policía Nacional es beneficiaria de un cúmulo de conocimiento transferido, una mejoría de capacidades sociales en su talento humano, amplitud de conocimiento en materia de Derechos Humanos y mejores capacidades en la resolución de conflictos, con una concientización e interiorización de la responsabilidad colectiva como parte del servicio social encabezado por el personal uniformado.

De igual manera, en el conjunto de beneficios personales se priorizan varios componentes para la vida personal y profesional de los uniformados que participan en las Misiones de Paz de la OnU, como la adquisición de mejores capacidades profesionales; conocimientos multiculturales que permiten mayores niveles de respeto y tolerancia hacia las distintas creencias; experiencia humanitaria, la cual lleva a una mayor humanización con las víctimas; implementación de nuevas estrategias sociales de intervención en comunidades necesitadas y un amplio conocimiento de los canales humanitarios existentes en Colombia y en el ámbito internacional para la atención de las víctimas.

En este sentido, la articulación semántica priorizada en cada uno de los conjuntos permite determinar la existencia de una acervo considerable de conocimiento adquirido con la capacidad de influir positivamente en los distintos roles que pueda cumplir la Policía Nacional en el marco del posconflicto en Colombia. 
Al realizar un comparativo de las capacidades y los conocimientos aportados y adquiridos por los uniformados de la Policía Nacional que han participado en la Misiones de Paz de la ONu (figura 2), se logró revelar de manera clara que existe una transferencia recíproca de conocimiento y capacidades entre la Policía Nacional y la ONU como resultado de la interacción de sus integrantes en las funciones que les han sido asignadas en los distintos países donde han estado.

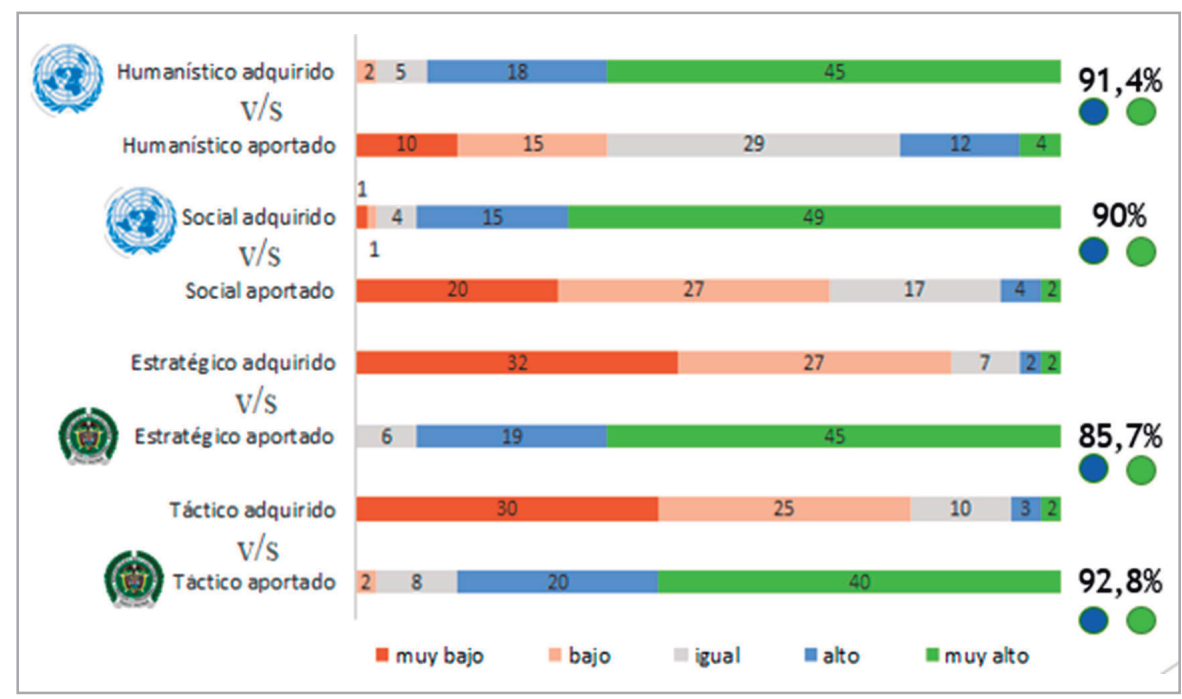

Figura 2. Paralelo de los conocimientos y las capacidades adquiridas y aportadas por la Policía Nacional en las Misiones de Paz de la ONU.

Fuente: Elaboración propia.

Como se observa en la figura 2, el 91,4 \% de los encuestados afirman que el conocimiento adquirido en el componente social es de un nivel alto y muy alto, mientras que el componente humanístico adquirido en los niveles alto y muy alto tiene un porcentaje del 90 \% de afirmación. En cuanto al conocimiento y las habilidades que aportan los uniformados de la Policía Nacional a las Misiones de Paz de la Onu, se estableció que los componentes tácticos y estratégicos desempeñan un papel fundamental en los despliegues de los uniformados de la Policía Nacional, puesto que sus niveles son altos en táctica $(85,7 \%)$ y muy altos en el componente operacional $(92,8 \%)$. 
Al evaluar el nivel de capacidades y conocimiento (figura 3) que adquieren los uniformados de la Policía Nacional como beneficio de su participación en las Misiones de Paz de la OnU, se encontró que estos alcanzan altos estándares de conocimiento en los componentes de asistencia humanitaria, proyectos productivos, responsabilidad colectiva, justicia social y Derechos Humanos. Este último se destaca en gran medida al ser elegido por el $100 \%$ de los encuestados como el componente de mayor impacto social en las labores realizadas por los uniformados en su trabajo con las víctimas de la violencia en las distintas comunidades intervenidas.

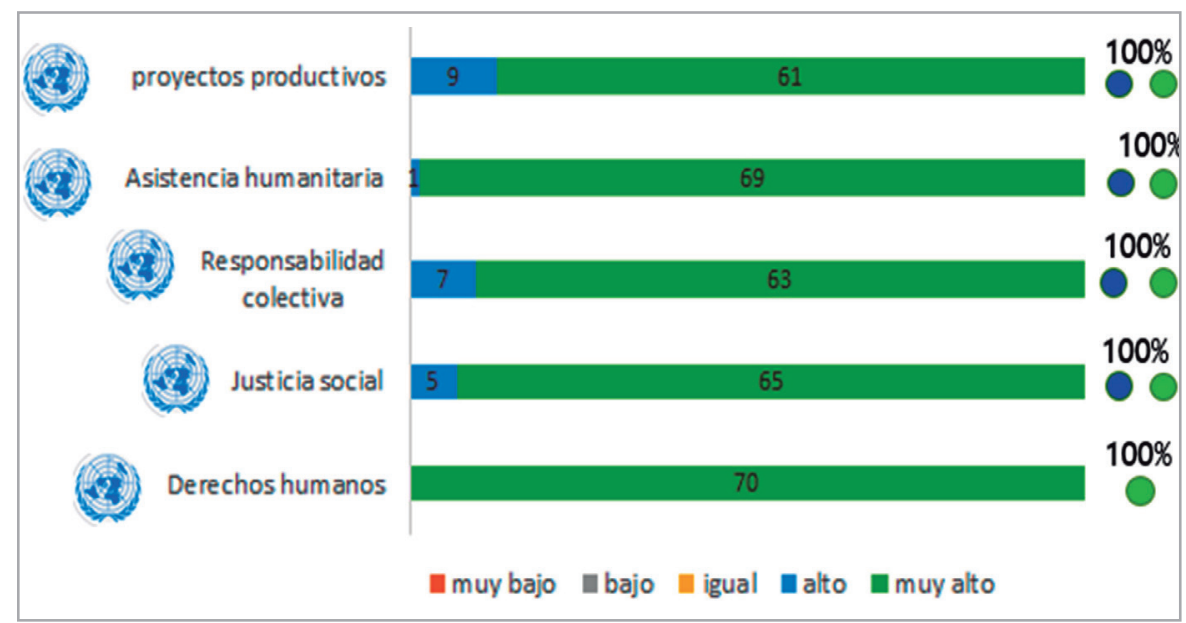

Figura 3. Nivel de conocimiento adquirido por la Policía Nacional en las Misiones de Paz de la ONU.

Fuente: Elaboración propia.

Teniendo en cuenta lo anterior, es importante señalar que la ampliación y el fortalecimiento del conocimiento en Derechos Humanos es una base fundamental de la formación de los uniformados de la Policía Nacional como garantes de derechos y libertades a todos los ciudadanos nacionales y extranjeros, sin matices de raza, religión, inclinación política o sexual, etc. 


\section{Discusión}

El contraste entre los insumos documentales y las herramientas de tipo experimental que se emplearon evidencia que la participación de la Policía Nacional en las Misiones de Paz de la Onu tiene la capacidad de hacer valiosos aportes al proceso de posconflicto en Colombia, a través del acervo histórico de conocimiento y habilidades que han sido adquiridas por la institución durante los 28 ańos que ha participado en diferentes intervenciones internacionales y en una gran variedad de conflictos armados.

En esta línea de ideas, cabe resaltar que la articulación semántica permitió descubrir que existen cuatro bases fundamentales que sustentan los beneficios que otorgan las Misiones de Paz de la OnU a los países donde tienen presencia y a los que participan en ellas mediante el despliegue de personal uniformado en procesos de cooperación. Estas bases fundamentales se sintetizan en los beneficios del ámbito internacional, nacional, institucional y personal, los cuales articulan la adquisición de conocimiento, estrategias y mecanismos necesarios para el desarrollo económico, político y social de cualquier país.

En el ámbito internacional se encontró que las Misiones de Paz tienen la capacidad de influir y mejorar las condiciones de paz, seguridad y calidad de vida, mediante el despliegue de programas sociales que incentiven el desarrollo social de los ciudadanos en los países donde se desarrollan las misiones. Asimismo, la información sobre el ámbito nacional evidencia que Colombia ha logrado establecer, mantener y fortalecer lazos de cooperación recíproca con otros países, al igual que el reconocimiento internacional de sus uniformados gracias a las capacidades que han demostrado en su participación en las Misiones de Paz de la OnU.

De otra parte, el ámbito institucional ha expuesto el mejoramiento de las capacidades humanitarias y en la resolución de conflictos de los uniformados, al igual que el aumento de sus capacidades de talento humano debido a la transferencia de conocimiento que tiene lugar cuando participan en las Misiones de Paz. Respecto al ámbito personal, se destaca la importancia que tiene para los uniformados de la Policía Nacional participar en las Misiones de Paz de la Onu, puesto que estas les aportan valiosas capacidades personales y 
profesionales mediante el contacto con otras culturas y las estrategias sociales que ofrecen los canales humanitarios.

Finalmente, es importante dejar claro que la participación de la Policía Nacional en las Misiones Paz de la Onu ha logrado influir en diferentes ámbitos de manera positiva, y también ha permitido una transferencia recíproca constante de conocimiento y capacidades entre la Policía Nacional y los organismos multilaterales, al igual que con policías de otros países. De igual forma, esta participación ha promovido la adquisición y el fortalecimiento de altos niveles de conocimiento en distintos componentes sociales, entre los cuales se destacan los Derechos Humanos como base social para fomentar las garantías de igualdad a todos los ciudadanos para su libre desarrollo político, sexual, religioso y social. Es en este escenario donde la Policía Nacional tiene un papel fundamental como vigilante, garante y defensor de los Derechos Humanos en el marco del posconflicto en Colombia en pro de una transformación social.

\section{Conclusiones}

Para finalizar esta investigación, se concluye que el acervo bibliográfico contenido en este documento provee un robusto contenido teórico e histórico de la importancia que tienen las Misiones de Paz de la Onu para el desarrollo humano, económico y social de las víctimas del conflicto. Asimismo, se deben destacar las cuatro bases sociales en diferentes ámbitos (internacional, nacional, institucional y personal) que fundamentan la importancia que tiene para los países participar en estas misiones.

En cuanto a la Policía Nacional, específicamente, se concluye que la institución aporta a las Misiones de Paz de la ONU un alto nivel de conocimiento táctico y estratégico, gracias a la experiencia que han adquirido sus uniformados en la lucha contra el terrorismo, el narcotráfico y la delincuencia organizada. De igual manera, la Policía Nacional adquiere un alto nivel de conocimiento social y humanístico (Derechos Humanos y ayuda humanitaria) como resultado de su participación en dichas misiones, lo cual le ofrece los instrumentos apropiados para intervenir de manera adecuada en las distintas etapas del posconflicto en Colombia. 
Finalmente, se recomienda no solo continuar con la participación de la Policía Nacional en las Misiones de Paz de la ONU, sino además incrementar la cantidad de uniformados que se integrarán en las futuras misiones con el propósito de aprovechar en mayor medida los beneficios que se adquieren en los diferentes ámbitos con la cooperación internacional.

\section{Referencias}

Álvarez, C. A. (2019). Análisis de la cooperación y participación de la Policía Nacional en Operaciones de Mantenimiento de la Paz ante las Naciones Unidas. Escuela de Posgrados de Policía Nacional.

Babbie, E. (2000). Fundamentos de la investigación social. Internacional Thomson Editores.

Boutros-Ghali, B. (1993). Maintaining international peace and security. Organización de las Naciones Unidas.

Fajardo, E. (2017). Hacia la caracterización de los valores democráticos y ciudadanos de los estudiantes universitarios: Una mirada desde la formación política y la construcción de escenarios de paz. Revista Interamericana de Educación, Pedagogía y Estudios Culturales, 9(1), 87-105. https://revistas.usantotomas.edu.co/index.php/riiep/article/ view/3604/3499

Kaufman, A. M., \& Rodríguez, M. E. (1993). La escuela y los textos. Santillana.

Lombana, J., Amashta, Y., Correa, C., \& Rodríguez, M. C. (2018). Benchmarking y análisis de competitividad de las cadenas productivas de quinua en Colombia, Perú y Bolivia. FACE: Revista de la Facultad de Ciencias Económicas y Empresariales, 17(2), 157-16. https:// doi.org/10.24054/01204211.v2.n2.2017.2891

Morales, O. (2016). Fundamentos de la investigación documental. Universidad de los Andes.

Organización de las Naciones Unidas [ONU]. (2008). United Nations Peacekeeping Operations Principles and Guidelines. Naciones Unidas.

Organización de las Naciones Unidas [ONU]. (2016). Informe del Secretario General sobre la labor policial de las Naciones Unidas. Organización de las Naciones Unidas (Consejo de Seguridad).

Organización de las Naciones Unidas [ONU]. (s. f.). Carta de las Naciones Unidas. Organización de las Naciones Unidas.

Pastrana, E. (2019). Fuerzas Militares de Colombia: Nuevos roles y desafios nacionales e internacionales. Hubert Gehring Editores.

Policía Nacional. (2010). Manual de Cooperación Internacional de la Policía Nacional de Colombia (t. 2). Policía Nacional.

Rodríguez, L. A. J., \& Suárez, R. G. (2017). Gestión del post-conflicto como estrategia de la responsabilidad social en las empresas. FACE: Revista de la Facultad de Ciencias Económicas y Empresariales, 17(1), 35-43.

Vallejo Almeida, G. (2015). Las operaciones de paz de la ONU, una opción para el caso colombiano. Universidad Católica de Colombia. https://repository.ucatolica.edu.co/bitstream/10983/14299/4/06_Las-operaciones-de-paz-de-la-ONU.pdf 